\title{
New stringent LIV limits from astrophysical gamma-ray sources
}

\author{
Rodrigo Guedes Lang, Humberto Martínez-Huerta and Vitor de Souza* \\ Instituto de Física de São Carlos, Universidade de São Paulo, Avenida Trabalhador \\ São-Carlense 400, CEP 13566-590, São Carlos, SP, Brazil \\ E-mail:vitor@ifsc.usp.br
}

\begin{abstract}
Lorentz invariance violation (LIV) introduced as a generic modification to particle dispersion relation can change the photon energy threshold of pair-production, which modifies the extragalactic background light (EBL) absorption of gamma rays from astrophysical sources. In this note, we report an innovative data analysis that allows us to extract unprecedented information from the most updated data set composed of 111 energy spectra of 38 different sources measured by current gamma-ray observatories. The outcome technique and results maximize the chances to find or discriminate LIV signals in any future search and reduce the biases in current analyses. Therefore, we report stringent limits for the LIV energy scale at first and second leading order in high energy gamma-rays. These limits are better than the ones current available in the literature for subluminal searches of LIV, irrespectively of many tested uncertainties, such as, poor knowledge of the EBL, large uncertainties in the intrinsic energy spectra functional form, energy resolution, selection of spectra and the energy bins selection used in the calculation of the intrinsic energy spectra.
\end{abstract}

36th International Cosmic Ray Conference - ICRC2019

24 July - 1 August, 2019

Madison, Wisconsin, USA

${ }^{*}$ Speaker. 


\section{Introduction}

The possibility to violate Lorentz invariance (LIV) has been proposed as a possible departure from the relativity principle [1]. The invariance of physics laws under Lorentz's transformation has been tested and restrictive limits have been imposed [2]. Possible invariances are proposed to be present in extreme phenomena and, thus, astroparticle physics is a suitable area to search for LIV signals.

LIV physics could result in an energy-dependent speed of light, vacuum Cherenkov, photon decay, and changes in the kinematics of interactions [3-5]. These effects could change astroparticle physics data in a detectable way. This work discusses the results obtained by Lang et al. [6] by searching subluminal LIV signals in the energy spectra of $\mathrm{TeV}$ gamma-ray sources. It is also shown preliminary results of the possibility of testing superluminal signal.

\section{Attenuation of gamma-rays including LIV}

Along the extragalactic path toward Earth, TeV gamma rays and EBL photons interact creating electron-positron pairs: $\gamma+\gamma_{E B L} \rightarrow e^{+}+e^{-}$[7]. Successive interactions attenuate the primary emitted gamma-ray flux as described by

$$
a(E, z)=e^{-\tau}(E, z)=\frac{J_{\text {meas }}(E)}{J_{\text {int }}(E, z)},
$$

where $J_{\text {meas }}$ is the measured spectrum at Earth and $J_{\text {int }}$ is the intrinsic spectrum emitted by the source. $a(E, z)$ is called attenuation and $\tau$ is the optical depth.

Under the LIV assumption, the energy threshold of the pair production is changed, and the attenuation modified by a change in the energy dispersion relation $[5,8]$. Changing the photon dispersion relation changes the energy threshold of the pair production:

$$
\varepsilon_{t h}^{\mathrm{LIV}}=\frac{m_{e}^{2}}{4 E_{\gamma} K(1-K)}-\frac{\delta_{n}^{\mathrm{tot}} E_{\gamma}^{n+1}}{4},
$$

where $\varepsilon_{t h}^{\mathrm{LIV}}$ is the background photon energy threshold considering LIV, $E_{\gamma}$ is the gamma-ray energy, $m_{e}$ is the electron mass, $K$ is the inelasticity, and $\delta_{n}^{\text {tot }}$ is a linear combination of the LIV coefficients from the different particle types, $\delta_{a, n}[4]$. Two cases are possible: subluminal $\left(\delta_{n}^{\text {tot }}<0\right)$ and superluminal $\left(\delta_{n}^{\text {tot }}>0\right)$ [9]. For simplicity, only LIV photons are considered and $\left|\delta_{n}\right|=1 /\left(\mathrm{E}_{\text {LIV }}^{(\mathrm{n})}\right)^{\mathrm{n}}$.

The subluminal case was discussed by Lang et al. [6]. Figure 1 shows the mean free path and the energy threshold of the superluminal case. The complete analysis of this effect in the detection of $\mathrm{TeV}$ gamma-rays is yet to be evaluated.

\section{LIV limits for the subluminal case}

The subluminal case was analyzed in detail in Lang et al. [6], and it is reviewed here. The analysis method was done in two steps (a) selection of the relevant measured spectra and (b) selection of the bins in each measured energy spectrum to be considered. This procedure minimized the systematic bias and resulted in a very robust result. 

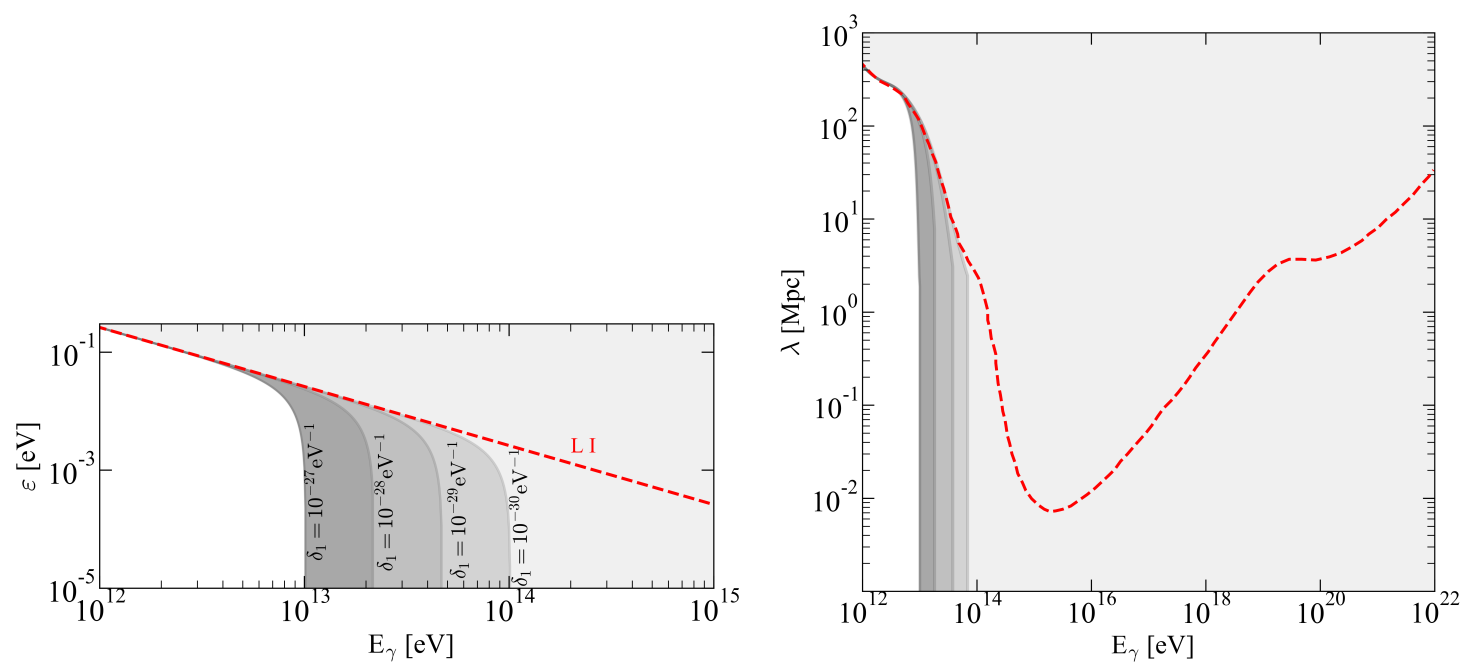

Figure 1: Mean free path for the pair production as a function of the photon energy. The red dashed continuous line represents the LI scenario. The left panel shows the background photon energy thresholds and the allowed pair production process configurations for the scenarios with the different LIV superluminal coefficients in the colored gray shades. The right panel shows the absolute mean free path for the same LIV scenarios, where the shaded regions are the none transparent universe as a function of the gamma-ray energy.

The method was applied to the most updated gamma-ray $\mathrm{TeV}$ data set. 111 measured energy spectra from 38 sources were analyzed from which 18 measured spectra from 6 sources were shown to contribute to restricting the LIV energy scale $\left(\mathrm{E}_{\mathrm{LIV}}^{(\mathrm{n})}\right)$ beyond the current limits. The data set is best described by the LI assumption. Therefore, LIV energy scale limits were imposed as shown in Tab. 1 and Figure 2. The 5 $\sigma$ CL limits, using the reference EBL model (Franceschini [10]), are 3.3 times better than the best limits in the literature for LIV subluminal signatures in high-energy gamma rays using $\mathrm{TeV}$ spectra analysis [11] and 3.6 times better than the best limits based on energy-dependent time delay [3]. For comparison, Figure 2 also shows previous strong exclusion limits to the LIV energy scale by similar subluminal analysis and the best limits based on energydependent time delay.

\begin{tabular}{l|ccc|ccc|ccc}
\hline \hline & \multicolumn{3}{|c|}{ Franceschini } & \multicolumn{3}{c|}{ Dominguez } & \multicolumn{3}{c}{ Gilmore } \\
\hline & $2 \sigma$ & $3 \sigma$ & $5 \sigma$ & $2 \sigma$ & $3 \sigma$ & $5 \sigma$ & $2 \sigma$ & $3 \sigma$ & $5 \sigma$ \\
\hline $\mathrm{E}_{\text {LIV }}^{(1)}\left[10^{28} \mathrm{eV}\right]$ & 12.08 & 9.14 & 5.73 & 6.85 & 5.62 & 4.17 & 14.89 & 9.80 & 4.74 \\
$\mathrm{E}_{\mathrm{LIV}}^{(2)}\left[10^{21} \mathrm{eV}\right]$ & 2.38 & 1.69 & 1.42 & 1.56 & 1.40 & 1.14 & 2.17 & 1.78 & 1.31 \\
\hline \hline
\end{tabular}

Table 1: Limits on the LIV energy scale imposed by this work using the EBL models Franceschini [10], Dominguez [12], and Gilmore [13].

\section{Acknowledgements}

We acknowledge the support from Fundação de Amparo à Pesquisa do Estado de São Paulo (FAPESP) through grants 2015/15897-1, 2016/24943-0, 2017/03680-3 and 2019/01653-4. We also acknowledge the National Laboratory for Scientific Computing (LNCC/MCTI, Brazil) for providing HPC resources of the SDumont supercomputer, which have contributed to the research results reported within this paper (sdumont.lncc.br). 

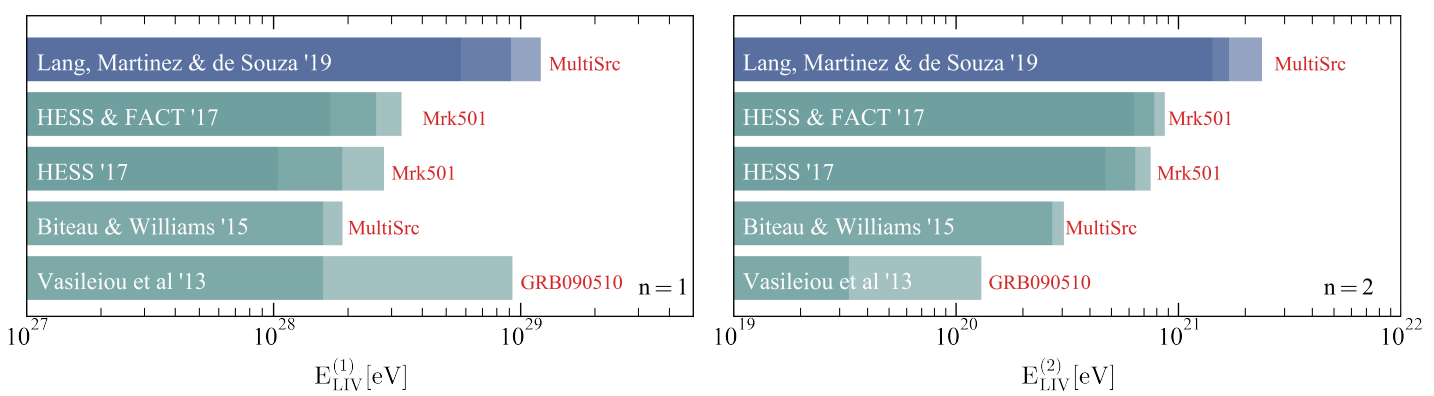

Figure 2: Comparison of the best limits imposed on the LIV energy scale. Left panel for $n=1$ and right panel for $n=2$. Shades of blue and green correspond to 2, 3, and $5 \sigma$ CL. Lang, Martínez and de Souza'19 (this work) and Biteau and Williams's 15 are based on multiple sources (MultiSrc), the latter of which are translated to the photon sector and the quadratic term. The other limits are based on specific measurements of one source as appointed. From top to bottom, see Refs. [6], [11], [14], [15], and [3].

\section{References}

[1] Mattingly D 2005 Living Rev. Rel. 85 (Preprint gr-qc/ 0502097 )

[2] Martínez-Huerta H 2019 Lorentz violation constraints with astroparticle physics 8th Meeting on CPT and Lorentz Symmetry (CPT'19) Bloomington, Indiana, USA, May 12-16, 2019 (Preprint $1906.06293)$

[3] Vasileiou V, Jacholkowska A, Piron F, Bolmont J, Couturier C, Granot J, Stecker F W, Cohen-Tanugi J and Longo F 2013 Phys. Rev. D87 122001 (Preprint 1305. 3463)

[4] Martínez-Huerta H and Pérez-Lorenzana A 2017 Phys. Rev. D95 063001 (Preprint 1610.00047 )

[5] Stecker F W 2003 Astropart. Phys. 20 85-90 (Preprint astro-ph/0308214)

[6] Lang R G, Martínez-Huerta H and de Souza V 2019 Phys. Rev. D99 043015 (Preprint 1810.13215 )

[7] De Angelis A, Galanti G and Roncadelli M 2013 Mon. Not. Roy. Astron. Soc. 432 3245-3249 (Preprint 1302.6460)

[8] Jacob U and Piran T 2008 Phys. Rev. D78 124010 (Preprint 0810.1318 )

[9] Martínez-Huerta H, Lang R G and de Souza V 2018 PoS BHCB2018 010 (Preprint 1901.03205 )

[10] Franceschini A, Rodighiero G and Vaccari M 2008 Astron. Astrophys. 487837 (Preprint $0805.1841)$

[11] Cologna G et al. (FACT, H.E.S.S.) 2017 AIP Conf. Proc. 1792050019 (Preprint 1611.03983 )

[12] Dominguez A et al. 2011 Mon. Not. Roy. Astron. Soc. 4102556 (Preprint 1007. 14 59)

[13] Gilmore R C, Somerville R S, Primack J R and Dominguez A 2012 Mon. Not. Roy. Astron. Soc. 422 3189 (Preprint 1104.0671 )

[14] Abdalla H et al. (H.E.S.S.) 2019 Astrophys. J. 87093 (Preprint 1901.05209 )

[15] Biteau J and Williams D A 2015 Astrophys. J. 81260 (Preprint 1502.04166) 